Tequio 1(1), 2017: 57-62

ISSN: 2594-0546

\title{
REFLEXIONES DE INCLUSIÓN EDUCATIVA: UN ESTUDIO CON FAMILIAS DE RETORNO
}

\author{
María de Jesús Pasallo-Zepeda ${ }^{1}$ \\ Ana María Méndez-Puga² \\ Fecha de recepción: 4 de noviembre de 2016 \\ Fecha de aceptación: 25 de mayo de 2017
}

\begin{abstract}
Resumen - La difícil situación económica por la que atraviesan familias mexicanas, las consecuencias de las políticas antimigratorias y la crisis económica de Estados Unidos han ocasionado cambios en los patrones migratorios. El incremento paulatino, durante los últimos años, de población deportada se vincula al aumento de grupos de personas interesadas en la educación de sus hijos. El presente artículo busca proponer ejes de análisis y reflexión para promover la inclusión escolar de niños migrantes de retorno, que se derivan del estudio cualitativo desde el enfoque de la etnografía en el que se trabajó con 15 menores, 10 padres de familia y 18 profesores. Los resultados muestran que los niños retornados están generando conocimientos que les permiten unir lo de aquí y lo de allá, pero la escuela no es consciente de ello, de ahí que se pretenda mostrar algunas de las reflexiones que esa dinámica motivó.
\end{abstract}

\section{Palabras clave:}

Educación, familia, inclusión educativa, infancia, migración de retorno.
Abstract - The difficult economic situation that cross Mexican families, the consequences of anti-immigrant policies and the economic crisis in the United States have caused changes in migratory patterns. The gradual increase, during the last years of returnees is linked to the increase of groups of people interested in the education of their children. This article seeks to create axes of analysis and reflection to promote the school inclusion of migrant children's return, same that are derived from the qualitative study, from the perspective of Ethnography in which they worked with fifteen return migrant children, ten parents and eighteen professors. The results show that returning children, across borders, are generating knowledge that enable them to join the here and there, but school is not aware of this, so that is intended to show some of the reflections generated by this dynamic.

\section{Keywords:}

Family, educative inclusion, childhood, education, return migration. 


\section{Introducción}

a migración se ha convertido en un suceso cada vez más frecuente. Específicamente Michoacánes uno de los estados de la República mexicana que registra mayores índices al respecto. De acuerdo con el Consejo Nacional de Población, aproximadamente uno de cada 11 mexicanos actualmente vive en el país del norte (Conapo, 2012); particularmente en lo que respecta a la entidad michoacana, se habla de 1.5 millones de personas que han salido hacia Estados Unidos (INEGI, 2010).

Ahora bien, de la misma manera que los compatriotas migran al país vecino, hay una gran población que está volviendo; es decir, regresando a sus lugares de origen. Por lo tanto, es posible afirmar que el retorno se convierte en una fase complementaria de la migración. Las estadísticas proporcionadas por el Instituto Nacional de Migración hablan de 4391898 repatriados de enero a noviembre de 2010, de los cuales 19296 son menores de edad, lo que equivaldría a 1754 niños al mes. Cabe aclarar que estas cifras no corresponden al porcentaje total de la población retornada, pues la repatriación se vincula más con términos legales, refiriéndose a quienes fueron deportados por autoridades migratorias, por lo que se trata de un regreso forzado. Se desconoce, por lo tanto, la cantidad de personas que volvió a México por decisión propia. Sin embargo, ambos casos (repatriación y retorno) tienen que ver con el regreso a sus comunidades de origen (Oller y Colomé, 2010, p. 201).

Este incremento paulatino de población retornada se asocia a la incorporación de contingentes significativos al medio educativo. La situación adquiere dimensiones preocupantes, sobre todosi se considera que prácticamente no existen datos que sean definitivos y confiables acerca de la magnitud y características del impacto que tiene la migración de retorno en la educación. Por este motivo es que surge mayor interés en el tema, ya que la escuela es el primer espacio de contacto sistemático que tienen las niñas y los niños con los nuevos modelos culturales, es ahí donde se les brinda una nueva manera de pensar, actuar, construir y reconstruir su presente.
Del anterior contexto es que se desprende la presente investigación, cuyo objetivo principal es proponer ejes de análisis y reflexión para promover la inclusión escolar de niños con esta característica. La indagación se basa en el paradigma cualitativo, desde el enfoque de la etnografía escolar. Participaron de forma voluntaria 15 niños migrantes de retorno, 10 padres de familia y 18 profesores; de los resultados es que derivan las reflexiones que a continuación se exponen. Cabe señalar que este trabajo es parte de una tesis de maestría titulada La construcción de la escuela como un espacio transnacional (Pasallo, 2014).

\section{La escuela inclusiva}

Bajo la lógica de esta investigación, la escuela es una institución formadora, facultada para actuar de manera responsable ante todas las demandas, que no debe ni puede limitarse al estilo de educación tradicional; asimismo, se espera que los docentes estén conscientes y capacitados acerca de nuevos y mejores métodos de enseñar, considerando situaciones como la llegada de niños migrantes de retorno a las aulas, donde se atienda aceptablemente las necesidades reales y actuales de los alumnos, favoreciendo la inclusión escolar del niño retornado equitativa y justamente, a fin de que pueda aprovechar las ventajas de ambas realidades y de ambas culturas.

Un aspecto detectado es la peculiaridad de algunas condiciones de vida de estos niños, en particular, las dificultades para aprender a la par que los demás, mismas que son consecuencia del desconocimiento de la lengua, de la nueva situación que enfrentan, incluyendo el cambio en todos los aspectos: académico, cultural y social, por lo tanto es necesario que el entorno escolar les ofrezca retos alcanzables, en un marco de respeto, apoyo y compresión (Sañudo, 2007). Oller y Colomé (2010) plantean que estos menores necesitan un tiempo de adaptación, ya que cada alumno vive una realidad distinta que es determinante a la hora de afrontar el reto académico con garantías; sin embargo, las diferencias no son un obstáculo para 
el avance escolar si se atienden adecuadamente las características de estos grupos.

Por otro lado, estas realidades escolares han implicado demandas no sólo para las y los profesores, sino también para el sistema educativo en general, en tanto que los niños que acceden a la escuela ya no son monolingües. De este modo, los contextos permeados por la migración obligarían a las instituciones escolares y a los docentes que laboran en ellas a aplicar nuevos sistemas de enseñanza; en pocas palabras, exige un cambio en la práctica educativa y docente. El binomio migración-educación formula retos a los cuales se está enfrentando la estructura educativa en México, algunos de los cuales ni siquiera son visualizados por los centros educativos, o bien son percibidos pero no se tiene el conocimiento suficiente para afrontarlos, lo que finaliza en la invisibilización.

Sánchez (2007) menciona que los nuevos profesionales de la educación y equipos docentes deberían basar su competencia profesional, en lugar de en su destreza para el uso de métodos específicos e individuales medidos por las características diferenciales de sujetos y grupos homogéneos, en su capacidad para ordenar un continuo de respuestas educativas de calidad adaptadas a las diferentes necesidades que manifieste todo el alumnado, en el que la diversidad de aportaciones de cada uno se convierta en medio y recurso para el logro de aprendizajes significativos. Como bien afirman Etxeberria y Elosegi (2010), lo idóneo sería un profesional capaz de conjugar los conocimientos con las actitudes necesarias para crear ambientes propicios para aprender, un profesor con la habilidad de diagnosticar la situación del aula, el ritmo y modos de aprendizaje de cada alumno, las características del proceso de aprendizaje, y que al mismo tiempo sepa cómo diseñar y planificar la enseñanza, sin olvidar que en el ámbito del aula se ha de procurar el equilibrio entre la comprensión del currículum y la atención a las diferencias individuales.

Sin embargo, la realidad arroja limitantes latentes en la práctica docente; en lo que concierne a la formación de los profesores, no existen espacios curriculares en los planes de estudio de las escuelas normales en México que destaquen la atención a escolares migrantes. Se requiere analizar este fenómeno para su entendimiento y tratamiento pedagógico en la formación, capacitación, actualización y especialización de los maestros mexicanos y estadounidenses. Del mismo modo, no es suficiente el cambio en las concepciones y la práctica de los docentes, es fundamental que la gestión de la institución apoye, propicie y consolide este modelo.

Es necesario mirar al alumno retornado bajo "el lente de la diversidad", a fin de favorecer que se sienta contento en la escuela, que perciba que está aprendiendo y que lo que aprende le servirá en su vida personal y profesional, además de que sus saberes son reconocidos. Bajo una lógica de educación inclusiva, este es el camino para la equidad; la escuela no debe regirse por la idea de que todos son iguales, al contrario, se deben reconocer y aprovechar las diferencias para que haya un mutuo enriquecimiento.

Se habla, pues, de una escuela inclusiva, y esto implica transformar el espacio educativo en un lugar para todos y todas, porque parte del principio de pertenencia al grupo y del aprendizaje entre todos. Los contenidos son comunes a la totalidad del grupo y se incorporan algunos específicos que respondan a las particularidades propias de cada uno, pero los contenidos comunes cada quien se los apropiará desde sus posibilidades, teniendo que disponer de múltiples momentos de intercambio. Igualmente, se flexibiliza el tipo de agrupación en función de las necesidades de la actividad. El enfoque, entonces, no es tratar de funcionar de la mejor manera o sólo buscar acomodar la escuela; se debe trabajar basados en la flexibilidad y el fomento de la comprensión de las diferencias individuales, considerando las variables personales del propio alumno. Este debe ser un proceso dinámico que se adapte constantemente, de acuerdo con las variaciones de las condiciones de cada niño o niña.

En esa educación inclusiva se deben abordar tres dimensiones, principalmente: la cultura, las políticas 


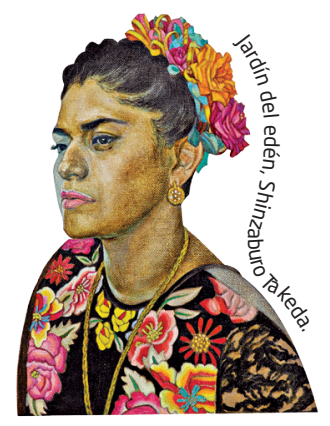

y las prácticas. Una cultura inclusiva supone construir una comunidad que cuida y valora a todos y a todo. El fomento de una política de inclusión demanda, en primer lugar, incentivar esa cultura y crear los mecanismos para dar respuesta a la diversidad del alumnado y asegurar su aprendizaje y participación, propiciando el desarrollo de mejores estrategias. Se busca que las actividades y prácticas en el aula y fuera de ella promuevan la participación y se tenga en cuenta su experiencia y conocimiento adquirido, tanto en la escuela como en otros ámbitos. De tal suerte que la escuela, vista desde y para todos, conduce a asumir que se debe construir saber junto con los niños en las aulas y fuera de ellas, considerando las diferencias, la curiosidad y el interés.

No se trata de separar el aula entre migrantes y no migrantes de retorno, y hacer el juego de la discriminación; más bien, hay que ser precavidos y buscar construir el conocimiento conjuntamente. Es posible partir de las diferencias entre los alumnos de un mismo salón para generar conocimientos con base en las vivencias de los niños y de sus familiares. De lo que se trata, entonces, es de considerar los conocimientos de los menores, iniciar de esa heterogeneidad para con ello posicionar a los niños como actores en la creación de nuevos conocimientos. De este modo, las experiencias de los alumnos migrantes de retorno, sus saberes, capacidades y su identificación con dos culturas y dos naciones son oportunidades para edificar la base de aprendizajes.

Cabe mencionar que la escuela es también una posibilidad de cambio social. Desde esa postura, la atención que se brinda en ella debe estar guiada por la respuesta a la diversidad, y en la medida en que responda a las necesidades e intereses de todos los estudiantes o de los que aspiran a serlo, se garantizará el derecho humano (Sañudo, 2007). Las escuelas facilitan la construcción de quienes participan de ellas, posibilitan también identificarse con el otro y con colectivos diversos, desde el juego, la escucha, la espera, el compartir, el dialogar. Reconocer las distintas diversidades que son parte de la escuela asegura que se atiendan las necesidades, el respeto a las garantías individuales y transformar la institución junto con los aprendientes.

No obstante las buenas intenciones, el mayor problema para los alumnos migrantes de retorno es pasar inadvertidos y ser invisibles. Zúñiga (2003) afirma que se les ha invisibilizado, porque en su mayoría quienes regresan a las escuelas mexicanas son muy similares al resto del alumnado, pues su apariencia física, su apellido y su domicilio les permiten confundirse enteramente con los demás compañeros. Lo anterior se observó en la escuela participante, aunque en cierto modo también son muy visibles si la comunidad es pequeña, porque se sabe que vienen de fuera, que no pertenecen, lo que no asegura que sean imperceptibles sus necesidades.

\section{Comentarios de cierre}

Desde este panorama, se puede decir que la migración bajo un escenario educativo ha sido prácticamente ignorada en México. Los estudiantes migrantes de retorno tienen una existencia poco palpable para el sistema educativo. Esta falta de visión ha dado como resultado insensibilidad por parte de instituciones escolares en relación con estos alumnos; en la mayoría de los casos se carece de estrategias para atenderlos, lo cual demuestra nuevamente que no han sido suficientes las acciones emprendidas. A pesar de que 
se espera que la escuela -como institución- sea un espacio capaz de migrar con los niños que regresan de Estados Unidos y se incorporan a las escuelas mexicanas, o viceversa, no ha sido así porque tampoco los maestros han podido comprender que ser migrante de retorno es una forma de concebir el mundo, una manera transnacional, ni de aquí ni de allá, pero de ambos lados al mismo tiempo.

En esta investigación se hace hincapié para que los niños migrantes de retorno reciban un trato donde quepan sus conocimientos sociales y culturales, donde sean reconocidos por sus competencias y habilidades. Puesto que la escuela es una entidad que debe trabajar para la sociedad, pensando en eso debe generar prácticas educativas que se esfuercen por entender a los sujetos, que amplíe la mirada y se rompan fronteras sociales, nacionales e idiomáticas. Como resultado de esta reflexión, se proponen algunos puntos de análisis en torno al tema:

- La escuela debe ser promovida como una alternativa que permite crear espacios flexibles y abiertos; es decir, que no se circunscriben a un solo país, espacios que sobrepasan las fronteras y que a su vez posibilitan condiciones para aprovechar lo mejor de ambos contextos y lograr así un adecuado desarrollo y bienestar. Los alumnos migrantes de retorno se nutren y viven en la frontera y a través de ella, es a partir de ahí donde fluyen, negocian, se adaptan, crean nuevos modos de ser y pertenecer, uniendo saberes, tradiciones, costumbres y valores de allá y de acá. Integran, así, múltiples aprendizajes que les ayudan a construirse y reconstruirse. De ese modo, los niños definen su identidad en medio de espacios plurales, buscando su lugar entre dos mundos y de dos mundos son sus dimensiones materiales y simbólicas.

- Los niños migrantes tienen derecho a ingresar a las instituciones educativas y a que éstas sean accesibles tanto en aspectos administrativos como en los contenidos escolares, haciendo efectiva la educación en tanto un derecho y no tan sólo a manera de servicio. Es necesario que se desarrollen políticas educativas que formen profesorados y educadores reflexivos y críticos, capaces de implementar prácticas transformadoras y de impulsar relaciones dialógicas y solidarias. Para para un mejor ejercicio de los planteamientos de la educación para todos, creemos que es indispensable contar con un profesorado calificado, que posea competencia pedagógica y muestre actitudes positivas que favorezcan la inclusión de todo el alumnado.

- Se requiere formular propuestas binacionales que aspiren a reestructurar los planes y programas curriculares, de tal forma que se mantengan la coherencia y continuidad entre los programas de ambos sistemas educativos.

- Las percepciones de los profesores y sus intentos por explicar las situaciones que se viven en el salón de clases a partir de la llegada de un alumno migrante de retorno, van unidos a sus experiencias y conocimientos individuales, mismos que parecen incentivar la construcción de conceptos clasificatorios que surgen de cierto conocimiento de la realidad, aunque estén poco fundamentados. Estos conceptos pueden convertirse con cierta facilidad en nuevos estereotipos o prejuicios, que al ser generalizados abanderan un tipo de práctica docente ineficaz para los niños que día con día llegan a los salones de clases mexicanos.

A partir de lo anterior se concluye que es urgente establecer una campaña de difusión de grandes alcances acerca de la migración como fenómeno social vigente y relevante, así como acerca de sus repercusiones en el ámbito educativo. Esta difusión debe hacer énfasis en la realidad multicultural y la necesidad de proveer condiciones para mejorar la calidad del servicio educativo que reciben los menores migrantes de retorno, además de en la necesidad de construir una educación inclusiva. Así, pues, es necesario realizar un trabajo en equipo 
entre las diferentes autoridades de la Secretaría de Educación, para buscar las estrategias que favorezcan la inclusión de los alumnos migrantes a los planteles, donde se recuperen y sistematicen las experiencias de los profesores con el trabajo de niños migrantes, compartiéndolas con otros docentes, a fin de manejarlas como un recurso. De igual modo, se necesita incorporar las vivencias de los menores que regresan en las actividades curriculares como elemento que enriquezca la comprensión de la diversidad bajo una escuela para todos y todas.

Finalmente, es importante reconocer que estos tiempos son de cambios educacionales y uno de los mayores retos es dar respuesta tanto a necesidades como a expectativas de las alteridades que arroja la migración en las prácticas educativas; y bajo el lema constitucional mexicano, que dice que "la educación debe ser de calidad y para todos", pensamos que el "para todos" engloba a los niños que se integran día con día a las aulas, los niños "nuevos", los "retornados", los "repatriados". De modo que estamos obligados a realizar esfuerzos en donde confluyan prácticas y compromisos de diversos sectores: políticos, centros de formación, directivos, familias y agentes sociales.

\section{Referencias}

Conapo (Consejo Nacional de Población). (2012). Índices de intensidad migratoria México-Estados Unidos 2010. Recuperado de: http://www.conapo. gob.mx
Etxeberria, F. y Elosegi, K. (2010). Nuevos retos en la escuela con inmigrantes. Revista Digital Ikastaria, 17(1), 67-89. Recuperado de http://www. euskomedia.org/PDFAnlt/ikas/17/17067089.pdf

INEGI. (2010). Estadística. Recuperado de http:// www.inegi.org.mx/est/contenidos/proyectos/ estadistica/default.aspx

Oller, C. y Colomé, E. (2010). Alumnado de otras culturas. Barcelona: Graó.

Sañudo, L. (2007). De la incorporación a la inclusión. Un estudio de la cultura educativa. México: Paidós.

Sánchez, J. (2007). El retorno de menores migrantes a escuela de Nuevo León. Trayectorias escolares, identidades transnacionales, dinámicas de inclusiónexclusión y trabajo docente. (Disertación doctoral). Universidad Autónoma de Nuevo León, México.

Pasallo, M. (2014). La construcción de la escuela como un espacio transnacional (Tesis de maestría). Universidad Michoacana de San Nicolás de Hidalgo, Morelia, Michoacán, México.

Zúñiga, V. (2003). El debate en torno al fracaso escolar de los alumnos mexicanos y de origen mexicano en las escuelas de Estados Unidos. Revista Internacional de Derecho y Ciencias Sociales, 1(3), 115-130. 